\title{
On the mutual influence of local resistances in the distribution of ventilation air
}

\author{
Alexey Kashurkin* and Vitaly Prokhorov \\ Moscow State University of Civil Engineering, Yaroslavskoe shosse, 26, Moscow, 129337, Russia
}

\begin{abstract}
The article deals with various methods of reducing the interference of local resistances in ventilation networks, as well as the question of the uniformity of air distribution on the branches. A critical assessment of the method of coarsening calculations used in practice is given, which is expressed in the fact that the reference coefficients of local resistances are simply summed up without taking into account the influence of local resistances on each other. It is proposed to use rectifying devices as a method of reducing the interaction of local resistances. The method of determining the length of the honeycomb jet rectifier necessary for the alignment of the velocity field of the flow is given. The use of flow dividers for the uniform distribution of the air flow through the branches is also considered, the system with flow separators is calculated and its pressure losses are compared with a similar system without flow separators.
\end{abstract}

As an example, a rectangular air duct of cross section Bxh 500x300 and tees with a rectangular inset on the narrow side of the air duct with a cross section of 200x100 in an amount of 5 pieces with the required flow rate of $250 \mathrm{~m} 3 / \mathrm{h}$ each were adopted. To simplify the subsequent calculation, it was assumed that the duct walls are smooth, so friction pressure losses are not taken into account.

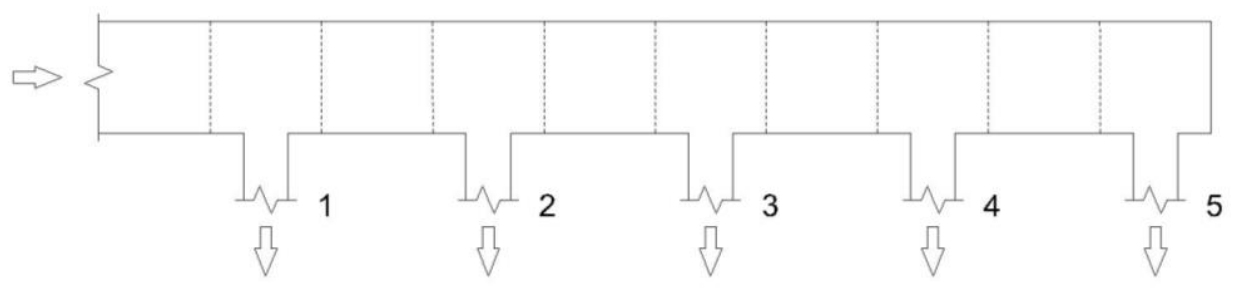

Fig. 1. Tees with a straight cut.

\footnotetext{
*Corresponding author: leontiii@mail.ru
} 
The calculation of a system with tees with a direct box (Fig. 1):

(a) Using the table (see diagram. 7-21 [1]), in relation to the area $\left(\frac{F_{n}}{F_{c}}\right.$ and $\left.\frac{F_{\sigma}}{F_{c}}\right)$ and in relation to the cost ( $\frac{L_{n}}{L_{c}}$ and $\left.\frac{L_{\sigma}}{L_{c}}\right)$ determine the value of the coefficient of local resistance tees "on the passage" (1-4) and "on the branch"(5), referred to the air velocity in the trunk before the branch:

$$
\zeta_{1}=0,55 ; \quad \zeta_{2}=0,52 ; \quad \zeta_{3}=0,5 ; \zeta_{4}=0,55 ; \quad \zeta_{5}=18,94 \text {. }
$$

(b) Calculate the velocity of the air flow in the trunk to the branch by the formula (1), $\mathrm{m} / \mathrm{s}$

$$
\begin{gathered}
v_{i}=\frac{L}{3600 \cdot f_{i}} \\
v_{1}=2,315 ; \quad v_{2}=1,852 ; \quad v_{3}=1,389 ; \quad v_{4}=0,926 ; \quad v_{5}=0,463 .
\end{gathered}
$$

(c) Determine the pressure loss by the formula (2), $\mathrm{Pa}$ :

$$
\begin{gathered}
\Delta P_{M c i}=\zeta_{i} \cdot \frac{\rho \cdot v_{i}^{2}}{2} \\
\Delta P_{M c 1}=1,769 ; \Delta P_{M c 2}=1,070 ; \Delta P_{M c 3}=0,579 ; \Delta P_{M c 4}=0,283 ; \Delta P_{M c 5}=2,436 .
\end{gathered}
$$

Total system pressure losses, Pa:

$$
\sum \Delta P_{M c i}=6,137 .
$$

Despite the relatively low values of pressure loss, it is necessary to remember that used in the calculation of reference values of coefficients of local resistances were obtained experimentally by the stable speed field and, moreover, under certain environmental conditions (particularly temperature and pressure) [2]. Even without trying to take into account the amendments to the changed external conditions, it should be understood that the distances between the following local resistances in practice, as a rule, are insufficient to stabilize the flow. For example, in [1] indicates that this requires a straight section with a length of up to 20 calibers, and in [4] this length after some local resistance should not be less than 100 calibers. The deformation of the flow velocity field leads to distortion of the local resistance coefficients in comparison with the reference data. At the same time, the total pressure loss of a system of several mutually influencing elements can be greater or less than the sum of the pressure loss of the isolated elements.

A logical way out of this situation can be an experimental determination of pressure losses for a group of local resistances, which, due to obvious reasons, may not always be feasible.

Another way to solve the problem of reducing the interference of local resistances can be the installation after each element of the network of rectifying the flow of devices. These can be various artificial obstacles evenly distributed over the cross section of the channel, such as grids and grids of different thickness and configuration.

Mention of the guide vane flow devices can be found in the works of domestic authors [1, p. 407], [4, p. 466], [5, p. 224-227] and [6, p. 72].

But this method has an obvious drawback: any additional element installed in the ventilation network leads to an increase in the cost of the entire system and has an additional coefficient of resistance. And the value of this coefficient for the mentioned 
means of equalizing the velocity field of the flow is the greater, the stronger its equalizing action, since the latter directly depends on the geometric parameters of this element (its thickness, geometric structure, area of the living section, etc.).

It should be noted that in addition to the unevenness of the flat velocity field in the flow, there are also vortex movements of air of different origin, which can be partially eliminated by means of a honeycomb jet rectifier (Fig. 2a).

In foreign literature, such devices are known as honeycomb air flow straightener and its straightening ability, in conjunction with the grids, in particular, has been studied in [8]

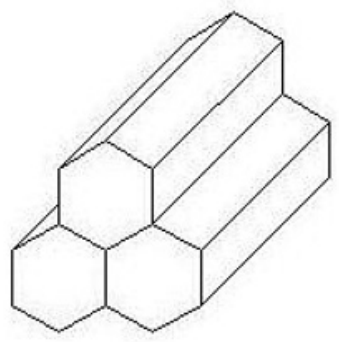

Fig. 2a. Fragment of a cellular jet straightener.

It's necessary geometric characteristics can be determined indirectly from the data $[1, \mathrm{p}$. 578], where, as mentioned above, the approximate values of the length of the straight section necessary to stabilize the flow are given. Thus, the length of the jet rectifier, which is necessary for sufficient stabilization of the flow at the outlet of it, should be equal to 1020 values of the hole width (in particular in [5, p.226] the length of the tubular jet rectifier is equal to 2 calibers, and in [8] tests at its length in 1 and 2 calibers showed comparable results). So, locally reducing the cross section for the passage of air, we get a compact device that satisfies the relative size of the flow stabilization condition.

It is worth noting that the excessive cost, as well as a significant increase in the total pressure loss of the system when using rectifying devices, lead to the fact that their practical application is not always advisable.

Also, when considering the system presented in figure 1, it should be understood that with such a configuration, the distribution of air on the branches will be to some extent different from the design.

This fact is in practice compensated by the installation on each of the branches of the control devices, which accordingly leads to an increase in their resistance and to a decrease in the influence of dynamic pressure in the main air duct.

Another way of uniformity of air distribution in branches is the use of tees with flow separators (Fig.3A). If in the previous embodiment, the air flow from the branches is due to the difference of static pressures in the duct and in the room, in this case, the flow occurs due to the difference of the total pressure in the separator and the static pressure in the room.

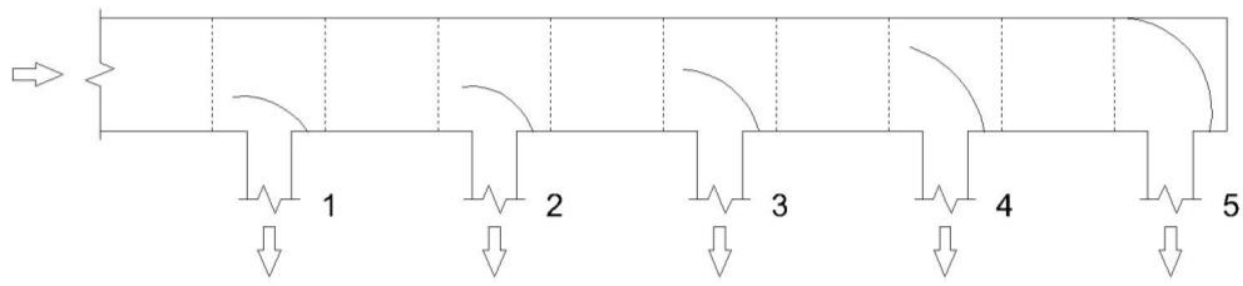

Fig.3a. Air distribution with flow separators. 
The flow separator is a flexible plate, the position of which in the duct is regulated by a simple graduated thrust (Fig. 3b).

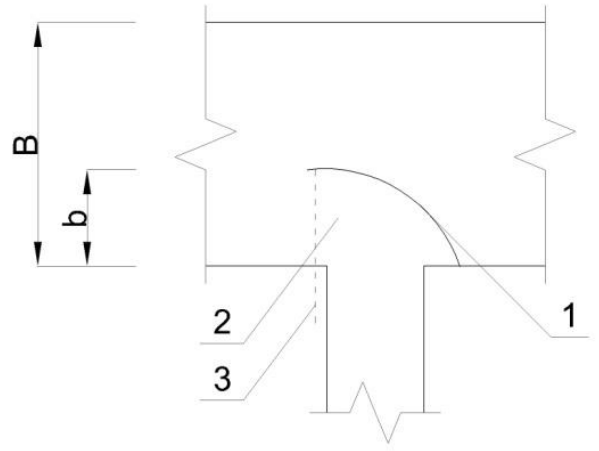

Fig. 3b. Scheme of a tee with a flow separator. 1. Flexible plate. 2. A shot of the separator flow. 3. A graduated rod.

In this case, the calculation is carried out according to the method described in [3]. In this paper, a system of 5 elements was experimentally investigated (a similar system is shown in Fig. 3a), but the use of the obtained dependences is possible with a different number of branches and different costs.

The calculation of a system with the separator flow (Fig. 3a):

a) According to the schedule (see Fig. 5, and [3]) find the relative sizes of the flow separators:

$$
\frac{b_{1}}{B}=0,315 . \quad \frac{b_{2}}{B}=0,4 ; \quad \frac{b_{3}}{B}=0,525 ; \quad \frac{b_{4}}{B}=0,725 ; \quad \frac{b_{5}}{B}=1 ;
$$

b) According to the schedule (see Fig. 5, b [3]) determine the coefficient of local resistance related to the velocity in the duct above the flow separator gate (1-4) and related to the velocity at the outlet of the hole (5):

$$
\zeta_{1}=1,25 ; \quad \zeta_{2}=1,5 ; \zeta_{3}=2,25 ; \quad \zeta_{4}=4,5 ; \zeta_{5}=0,9 \text {. }
$$

c) We calculate the absolute values of the alignments by the formula (3), mm:

$$
b_{i}=500 \cdot \frac{b_{i}}{B}
$$

$$
b_{1}=157,5 ; \quad b_{2}=200 ; \quad b_{3}=262,5 ; \quad b_{4}=362,5 ; \quad b_{5}=500 \text {; }
$$

d) We determine the area of the living section in the part of the air duct above the flow separator by the formula (4), $\mathrm{m}^{2}$ :

$$
f_{\text {cmвi }}=\left(B-b_{i}\right) \cdot h
$$

$$
f_{\text {cm } 1}=0,10275 ; \quad f_{\text {сmв } 2}=0,09 ; \quad f_{\text {сmв } 3}=0,07125 ; f_{\text {cmB } 4}=0,04125 \text {. }
$$

e) Calculate the velocity of the air flow in the duct above the gate separator and at the entrance to the last branch of the formula (5), $\mathrm{m} / \mathrm{s}$ :

$$
v_{i}=\frac{L}{3600 \cdot f_{i}}
$$




$$
v_{1}=2,703 ; \quad v_{2}=2,315 ; \quad v_{3}=1,949 ; \quad v_{4}=1,684 ; \quad v_{5}=3,472 \text {. }
$$

f) Determine the pressure loss by the formula (6), $\mathrm{Pa}$ :

$$
\begin{aligned}
& \Delta P_{M c i}=\zeta_{i} \cdot \frac{\rho \cdot v_{i}^{2}}{2} \\
& \Delta P_{M c 1}=5,480 ; \quad \Delta P_{M c 2}=4,823 ; \Delta P_{M c 3}=5,128 ; \Delta P_{M c 4}=7,657 ; \Delta P_{M c 5}=6,510 .
\end{aligned}
$$

Total system pressure losses, $\mathrm{Pa}$ :

$$
\Sigma \Delta P_{M C i}=29,598
$$

Although the total pressure loss in this case will be much higher, but the use of flow separators leads to the elimination of a significant drawback of the first system - the probable inaccuracy of the air flow distribution along the branches.

Conclusion: in connection with modern trends in energy saving, the question arises of further improvement of methods of aerodynamic calculation in terms of determining pressure losses in local resistances, taking into account their mutual influence.

\section{References}

1. Idelchik I. E. Handbook of hydraulic resistances / ed. M. O. Steinberg.- 3rd ed., pererab. I DOP. - M.: mechanical engineering, (1992)

2. Gorlin S. M., Slezinger I. I. Aeromechanical measurements (methods and devices) (M.: Nauka, 1964)

3. Prokhorov V. I. air ducts of uniform distribution with flow separators / / air Conditioning. A collection of 15. - M.: Gosstroiizdat, 1963, p. 179-190.

4. Transfiguration VP Thermal measurements and instruments. - Moscow: Energy, 1978.

5. Chistyakov S. F., Radun D. V. heat engineering measurements and devices. (Moscow: Higher school, 1972)

6. Kersten I. O. Aerodynamic tests of mine fan units. (M.: Nedra, 1986)

7. Taliev V. N. Aerodynamics of ventilation. (Moscow: Stroyizdat, 1979)

8. C.Farell, S.Youssef - Experiments on Turbulence Management Using Screens and Honeycombs, Journal of Fluids Engineering, vol. 118, pp. 26-32. (1996) 